http://doi.org/10.15359/ree.14-1.13

\title{
La investigación educativa interdisciplinaria integrada en el Centro de Investigación y Docencia en Educación (CIDE): un aporte de las Unidades Académicas y del INEINA
}

\author{
Magdalena Alfaro Rodríguez ${ }^{1}$ \\ Centro de Investigación y Docencia en Educación (CIDE), Universidad Nacional \\ Heredia, Costa Rica \\ Jorge R. Martín Pérez ${ }^{2}$ \\ Centro de Investigación y Docencia en Educación (CIDE), Universidad Nacional \\ Heredia, Costa Rica \\ Marie-Claire Vargas Dengo \\ Centro de Investigación y Docencia en Educación (CIDE), Universidad Nacional \\ Heredia, Costa Rica
}

Recibido 27 de octubre de 2009 • Aceptado 02 de diciembre de 2009

Resumen. El Proyecto Perfiles, Dinámicas y Desafíos de la Educación Costarricense en sus distintas etapas realiza investigación educativa en un nivel integrado del Centro de Investigación y Docencia en Educación (CIDE) de la Universidad Nacional en Costa Rica. Este artículo aborda la importancia de retomar los resultados y productos de tales investigaciones, en los distintos programas de formación docente que se ofrecen en este Centro.

Palabras clave. Educación, formación docente, investigación educativa, Centro de Investigación y Docencia en Educación (CIDE), Instituto de Estudios Interdisciplinarios de la Niñez y la Adolescencia (INEINA).

\footnotetext{
${ }^{1}$ Magíster en Educación con énfasis Docencia Universitaria y Licenciada en Música con énfasis en Educación Musical de la Universidad Nacional, Costa Rica. Actualmente, es académica de la División de Educología del Centro de Investigación y Docencia (CIDE) de la Universidad Nacional, Costa Rica, donde integra el equipo de investigación en la IV etapa del Proyecto Perfiles, Dinámicas y Desafíos de la Educación Costarricense. Correo electrónico magdar613@hotmail.com

${ }^{2}$ Magíster en Educación, Licenciado en Economía y Licenciado en Cibernética Matemática. Actualmente, es académico de la División de Educación para el Trabajo del Centro de Investigación y Docencia (CIDE) de la Universidad Nacional, Costa Rica, donde integra el equipo de investigación en la IV etapa del Proyecto Perfiles, Dinámicas y Desafíos de la Educación Costarricense. Correo electrónico: jorgeramonmartin@ yahoo.es

${ }^{3}$ Máster en Currículum e Instrucción de The George Washington University, USA. Actualmente, es académica de la División de Educación Básica del Centro de Investigación y Docencia (CIDE) de la Universidad Nacional, Costa Rica, donde integra el equipo de investigación en la IV etapa del Proyecto Perfiles, Dinámicas y Desafíos de la Educación Costarricense. Correo electrónico: educare@una.ac.cr
}

La Revista Electrónic@ Educare está indizada en el Catálogo LATINDEX.

Artículo protegido por la licencia Creative Commons. 
Abstract. The project called Perfiles, Dinámicas y Desafíos de la Educación Costarricense (Profiles, Dynamics and Challenges of the Costa Rican Education) in its various working phases it conducts interdisciplinary educational research at an integrated level at the Centro de Investigación y Docencia en Educación (CIDE) (Research and Teaching Center) from Universidad Nacional in Costa Rica. This article discusses the importance of taking into account outcomes and products of such research work within the various majors that CIDE offers.

Key words. Education, teaching education, educational research, Center of Research and Teaching in Education (CIDE), Institute of Children, Adolescence and Family Studies (INEINA).

\section{Introducción}

El momento histórico actual, caracterizado por constantes y progresivos cambios tales como: el desarrollo vertiginoso de la tecnología, la expansión del conocimiento, la globalización, el encuentro y el desencuentro de paradigmas contrapuestos, plantea para las escuelas, facultades y centros de formación docente el reto de pensar la educación para los años futuros, definir líneas de formación y preocuparse no sólo por cumplir con criterios preestablecidos de calidad en términos de eficiencia y eficacia, sino por responder a lo que la comunidad estudiantil y el complejo contexto cambiante demandan como urgencias cruciales emergentes.

La investigación que se realiza desde las escuelas de educación de las instituciones estatales de educación superior tiene pertinencia social, en tanto que logra identificar áreas y problemáticas sociales conducentes a generar oportunas respuestas educativas.

El propósito de este artículo es enfatizar sobre la relevancia de los resultados de la investigación educativa, que se realiza en un nivel interdisciplinario en el Centro de Investigación y Docencia en Educación (CIDE) de la Universidad Nacional, para los procesos de formación docente de las carreras que se ofrecen en este Centro y su incidencia en la sociedad costarricense.

\section{EI Centro de Investigación y Docencia en Educación (CIDE) y la investigación interdisciplinaria en un nivel integrado del Centro}

Durante la última década, y con el objetivo de constituirse en un referente nacional en materia educativa en los ámbitos nacional y latinoamericano, el Centro de Investigación y Docencia en Educación (CIDE) desarrolla procesos académicos y de investigación que fortalecen su quehacer en distintos ámbitos universitarios. En relación con estos procesos, debe mencionarse

el esfuerzo de investigación llevado a cabo por el CIDE, mediante sus distintas Unidades Académicas, que han abordado temáticas que aportan al conocimiento en el campo educativo y permiten fortalecer sus planes de estudio en la formación docente, en la actualización profesional y el mejor conocimiento acerca del sistema educativo costarricense. (Castro, Esquivel, González, Induni, Pereira, Solano et al, 2005, p. 4).

Asimismo, debe acotarse que, en el año 2002, se aprobó el Proyecto Perfiles, Dinámicas y Desafíos de la Educación Costarricense como parte del Programa Educación y Desarrollo en Costa Rica, adscrito al Decanato del CIDE, con el propósito de retomar "el quehacer en docencia, investigación, extensión y producción del CIDE que se sustente en la contribución de las diferentes unidades académicas y del Instituto 
de Estudios Interdisciplinarios de la Niñez y la Adolescencia (INEINA)" (Decanato-CIDE, 2005, p. 6).

El Proyecto Perfiles, Dinámicas y Desafíos de la Educación Costarricense, en sus distintas etapas, ha desarrollado investigación educativa en un nivel integrado del CIDE Actualmente, destaca la importancia que tiene para la formación docente en el país, retomar los productos y los resultados de las investigaciones del mencionado proyecto, con miras a contribuir al mejoramiento de la educación costarricense y, en particular, de la formación docente.

Cada etapa de investigación del proyecto tiene un valor particular, según se explica a continuación.

En la primera etapa, se realizó una exhaustiva y comprensiva investigación sobre la dinámica de la educación en Costa Rica, con el objetivo de

realizar un análisis de la situación de la educación en el país, a partir del reconocimiento de las principales dinámicas que tienen lugar en el sistema de educación formal, en los niveles de preescolar; I, II y III ciclos de la Educación General Básica y Educación Diversificada, enfatizando en cobertura y permanencia. (Castro et al., 2005, p. 1)

En esta investigación, se llevó a cabo una revisión de los principales aportes de investigaciones sobre educación en Costa Rica hasta la fecha de su ejecución y, también, se desarrolló un trabajo de investigación de campo en siete direcciones regionales del país, a saber: Desamparados, Upala, Belén, San Carlos, Limón, Nicoya y Pérez Zeledón. En la investigación, se realizó un análisis de información de esas regiones, con el claro propósito de indagar los aspectos que condicionan los procesos de éxito o de fracaso escolar en el país.

El estudio confirmó la existencia de brechas significativas entre las dependencias educativas y las diversas zonas. Además, aportó valiosa información sobre las especificidades de los contextos socio-económicos y educativos de las regiones en las que se llevó a cabo la investigación, e indicó que, en su mayoría, tienen un índice de desarrollo social muy bajo (Castro et al., 2005).

En la segunda etapa de investigación del Proyecto Perfiles se identificaron los factores de éxito que inciden en la difícil transición escolar de sexto a sétimo año y, por ende, en la permanencia en el III ciclo de la Educación General Básica.

Los resultados de las exploraciones realizadas durante esta etapa sobre factores de éxito en la transición de sexto a sétimo año, lograron identificar el papel de la y el docente como uno de los factores claves para la permanencia y el éxito escolar.

Entre otros hallazgos, los datos proporcionados por la población estudiantil para esta investigación arrojaron que: el 57,1\% muestra la existencia de un problema metodológico considerable, el cual afecta los procesos de enseñanza y de aprendizaje; el 44,4\% de los estudiantes de sétimo y un $52,02 \%$ de octavo, apuntan que la metodología y la habilidad de cada docente, para explicar, son las razones para justificar si un profesor es bueno; el 31,48\% expresa que es la relación personal (actitud de apoyo y refuerzo positivo) la que les lleva a tipificar sus docentes como los mejores; el 18,4\%, en sétimo, y el 19,1\%, en octavo, manifiestan que "el bajo nivel de comprensión de las materias básicas" es lo que les afecta mayormente cuando ingresan a sétimo (Castro, Díaz, Fonseca, León, Moraga, Ruiz et al., 2007, p. 181).

Por otra parte, los datos arrojados por los docentes entrevistados en la investigación, señalan la importancia de la preparación de los y las docentes que atienden a los y las estudiantes (Castro et al., 2007, p. 181).

En la tercera etapa de investigación del Proyecto y, con base en los resultados de las investigaciones anteriores del proyecto, se optó por indagar sobre el perfil del docente necesario 
para asumir un grupo de sétimo año. Para ello, se realizaron observaciones de campo conducentes a detectar aspectos del desempeño docente que favorecen la relación estudiante-profesor. Además de un proceso de recolección de información teórica-conceptual, se llevó a cabo un proceso investigativo a partir de entrevistas, cuestionarios y talleres, en instituciones de secundaria en distintas zonas del país, para identificar aquellos aspectos pertinentes al perfil del docente para sétimo año de la educación pública general básica costarricense. Como producto de esta investigación, se elaboró un perfil integrado emergente de desempeño docente para sétimo año de la educación pública costarricense (Alfaro, Jiménez, Martín, Ramírez \& Vargas, 2008b). Esta investigación fue de tipo cualitativo.

Actualmente, en la cuarta etapa, se replica la investigación de la tercera etapa para elaborar el perfil docente para el tercer ciclo completo de la educación pública costarricense. Esta investigación, como la anterior, es de carácter cualitativo y aporta, también, las características y las cualidades docentes que permiten un mayor acercamiento a los y las estudiantes en los niveles de formación del III ciclo, así como un mayor entendimiento de los y las jóvenes de este nivel educativo, según su madurez física y emocional, sus formas de interacción, su actuación, las distintas formas en que aprenden y el contexto en que se desenvuelven. Con ello, se procura delinear la selección de los perfiles docentes y propiciar la promoción estudiantil a los siguientes niveles escolares, así como su permanencia dentro del sistema educativo (Alfaro et al, 2008c).

Los procesos de las distintas investigaciones mencionadas reflejan la misión explícita del CIDE, en tanto que ésta estipula desarrollar procesos de investigación educativa y compromete su extensión con las comunidades, para impulsar el mejoramiento cualitativo y continuo de la educación. Paralelamente, se relacionan con la visión del Centro, la cual acota que la investigación (además de docencia, producción académica, divulgación del conocimiento) está comprometida con el derecho a la educación y con las necesidades educativas individuales de las diversas poblaciones del país (Decanato-CIDE, 2007).

Los productos resultados de las investigaciones del Proyecto Perfiles, Dinámicas y Desafíos de la Educación Costarricense, así como los hallazgos extraídos, pueden contribuir al mejoramiento de la educación costarricense, en el sentido de que al indagar distintos ámbitos educativos, proporcionan información sobre distintas comunidades nacionales, sus actores y sus instituciones educativas públicas. Esto permite comprender mejor su dinámica, sus necesidades y sus fortalezas, lo cual posibilita un acercamiento más oportuno y atinado desde las escuelas de formación docente y de los futuros y las futuras docentes.

La relevancia de la investigación educativa que se realiza desde las instituciones de educación superior y los aportes que ésta puede generar a la formación docente, sugieren considerar lo que Teichler (2003) refiere como importante para prever futuros problemas y temas de debate, a fin de desarrollar conceptos y generar conocimiento con suficiente antelación, pues si se espera, para abordar una problemática, sólo el momento cuando ésta es objeto de acalorados debates, difícilmente se puede ofrecer un conocimiento sistemático como resultado de investigación.

\section{Responsabilidad de los Centros y las Escuelas de formación docente}

La labor educativa en Costa Rica es un reto que implica replantearse y valorar el papel del educador y de la educadora en las escuelas, los colegios y las aulas universitarias. Este reto, asumido por las escuelas de formación docente del país, requiere el compromiso consciente para integrarse, responsablemente, a la gestión educativa, en sintonía con la dinámica global actual, los imperativos de preservar y fortalecer los intereses y los valores de la identidad nacional y de la 
sociedad costarricense.

Las polémicas actuales por la dignificación del rol docente no transcurren en el vacío, ni se resuelven en el plano discursivo, sino que deben ser contextualizadas. Esto significa que se debe considerar como indispensables los contextos social, regional, comunal e institucional, en los que se desempeñan los y las docentes, así como las características propias de la diversidad de la población estudiantil, según su desarrollo biopsicosocial, su estadio maduracional y las características personales en relación con el contexto social en que se desenvuelven.

El CIDE, como centro de formación docente de la Universidad necesaria es partícipe de lo que Dengo (1997) concibe como la función de largo alcance que han desempeñado las universidades públicas costarricenses, dentro del sistema educativo nacional y, a la vez, constituye, histórica y constitucionalmente, uno de los aportes más significativos de las instituciones de educación superior al sistema educativo.

En el nivel de Centro, la gestión académica y la gestión administrativa del CIDE se fundamentan en valores de humanismo, de excelencia, de responsabilidad social, de integridad, de equidad y de respeto a la diversidad. En el CIDE, la formación docente-profesional cobra significado y relevancia al enmarcarse dentro de un proyecto histórico-pedagógico que define el tipo de personaciudadano que se desea formar.

Los planteamientos de las políticas nacionales vigentes referentes al siglo XXI (CONARE, 2006), en materia educativa, sugieren un perfil de persona-ciudadano construido en relación con el momento histórico de la sociedad costarricense. Según el segundo Estado de la Educación Costarricense (2008), "la institución de educación superior que más se acerca al perfil derivado de los lineamientos de la política educativa es la UNA" (p. 145). En concordancia con lo anterior, para contribuir al mejoramiento de la educación costarricense "sólo del programa de la UNA se infiere que se busca un docente autónomo, con visión ilustrada y capacidad crítica" (CONARE/Programa Estado de la Nación, p. 145).

Lo anterior es válido tanto para los planes de estudio regulares como para las propuestas de educación continua, en tanto que la formación continua contribuye a mantener un perfil docente actualizado con la época presente. Esto se fundamenta en autores como Shön (1998), para quien las habilidades profesionales se construyen a partir de la experiencia, la cual significa "aprender en y mediante la práctica".

En correspondencia con esta posición, el equipo interdisciplinario de investigación del Proyecto Perfiles, Dinámicas y Desafíos de la Educación Costarricense, en su IV etapa, concuerda con que la formación docente va más allá de la formación inicial que se da en una primera etapa de formación profesional, pues no solamente se es profesional de la educación cuando se egresa de los estudios universitarios con una titulación, sino que esta formación se profesionaliza a medida que se entra en contacto con una institución educativa, se desempeña y se vive la labor docente.

\section{Responder a los cambios educativos de la sociedad costarricense}

Dado que es responsabilidad de las escuelas de formación docente de las universidades estatales costarricenses, configurar una acción formativa pertinente y eficaz que responda a las exigencias del contexto nacional actual, es necesario replantear, constantemente, la formación de maestros y profesores que se ofertan para el sistema educativo nacional en todos los niveles, tanto en zonas urbanas como rurales. Entre otros aspectos, esto tiene implicaciones urgentes para abordar, por ejemplo, las especificidades de la docencia que las instituciones de atención prioritaria requieren y, por 
tanto, revertirlas en los programas formativos. Este planteamiento alude, por ejemplo, a la importancia de retomar los resultados de investigación de un perfil académico-profesional de educador(a) actualizado(a) con la época y con los contextos social, nacional y global (Alfaro et al., 2008a).

En el marco de la pedagogía costarricense actual, la formación docente cobra especial interés y relevancia a causa de, entre otros aspectos, la necesidad de mejoramiento de la educación, la urgencia de solventar distintas situaciones de deserción, de rezago escolar y de realizar una actualización, de acuerdo con los cambios del entorno y del mundo. Pueden mencionarse varios ámbitos de actualización profesional relacionados con las innovaciones en el entorno global, entre ellos: ciencia, tecnología y medios de información. Los ámbitos de actualización, en relación con los entornos inmediato, nacional y comunal, requieren de preparación, conocimiento e información sobre aspectos culturales, económicos y sociales, emergentes en una comunidad específica, que determinan formas de comportamiento e interacción de la población estudiantil y que pueden intervenir en el rendimiento escolar.

La formación continua cobra relevancia para la actualización docente, en tanto que debe responder al momento presente para no estar educando a espaldas del tiempo y lejos de las demandas del entorno cambiante, lo que amenazaría con la obsolescencia de lo aprendido en el pasado, en los momentos de formación inicial (Alfaro, Gamboa, Jiménez, Martín, Ramírez \& Vargas, 2009). Los centros y escuelas de formación docente cumplen una función importante al ofrecer talleres y cursos de extensión que posibiliten esa formación.

El mejoramiento de la educación en los niveles de primaria y de secundaria está estrechamente ligado al tema de la formación docente y a la formación continua, pues aunque la oferta de carreras de formación docente en el país es prolífica, no por ello todas cumplen con criterios de calidad y de excelencia. Una revisión exhaustiva de esta formación contribuiría a responder, claramente, desde los centros de formación docente, a los cambios educativos que la sociedad costarricense del siglo XXI demanda.

La calidad de la educación no debería, entonces, consistir, únicamente, en aprobar procesos de acreditación de las distintas carreras docentes y cumplir con ciertos criterios, sino, sobre todo, en determinar las repercusiones y las contribuciones que a futuro puede lograr la formación docente en los distintos ámbitos educativos y sociales del país. Justifica esta necesidad el hecho de que, en la actualidad, Costa Rica enfrenta nuevas demandas y mayores exigencias sociales, tales como son el ampliar sus logros en desarrollo humano y el generar mayores oportunidades (Román, 2009).

\section{Conclusiones}

En las distintas etapas del Proyecto Perfiles, Dinámicas y Desafíos de la Educación Costarricense se ha desarrollado investigación educativa interdisciplinaria en un nivel integrado de Centro. En cada etapa, se ha recopilado gran cantidad de información, se ha elaborado y se ha producido conocimiento teórico-conceptual, como resultado de la investigación in situ en distintas regiones del país.

Es un reto institucional universitario plantear la educación necesaria para los próximos años, definir líneas de formación, vincular los resultados de investigación en los procesos de formación para atender no sólo las demandas de los procesos de acreditación, garantes de calidad, sino, también, atender lo que el contexto cambiante presenta como desafíos cruciales y que, de una forma $\mathrm{u}$ otra, las investigaciones logran detectar.

Por lo anterior, se concluye como aspecto importante el valorar los procesos y los resultados de la investigación educativa que se realiza en un nivel interdisciplinario del Centro de Investigación y Docencia en Educación, de la Universidad Nacional. Es relevante que se constituya en un referente nacional y latinoamericano en materia educativa y que se retomen y se aprovechen, en los distintos procesos de formación docente ofrecidos en las carreras regulares y en los programas de 
actualización y de formación continua, el cúmulo de conocimientos compilados y generados como producto de la investigación.

\section{Referencias bibliográficas}

Alfaro, M., Gamboa, A., Jiménez, S., Martín, J., Ramírez, A. \& Vargas, M. (2008a). Construcción del perfil profesional docente de sétimo año: respuesta a una necesidad actual. Revista Educare, Vol. XII, N ${ }^{\mathrm{o}} 2,31-45$.

Alfaro, M., Jiménez, S., Martín, J., Ramírez, A. \& Vargas, M. (2008b). El perfil integrado emergente de desempeño docente para sétimo año. Heredia, Costa Rica: CIDE, Universidad Nacional.

Alfaro, M., Gamboa, A., Jiménez, S., Martín, J., Ramírez, A. \& Vargas, M. (2008c). III Informe final: Proyecto Perfiles, Dinámicas y Desafíos de la Educación Costarricense. Heredia, Costa Rica: CIDE, Universidad Nacional.

Alfaro, M., Gamboa, A., Jiménez, S., Martín, J., Ramírez, A. \& Vargas, M. (2009). Formación docente, modelos y retos. Manuscrito presentado para su publicación.

Castro, M., Esquivel, L., González, A., Induni, G., Pereira, Z., Solano, J. et al. (2005). I Informe final de investigación. Perfiles, Dinámicas Y Desafíos del Sistema Educativo Formal Costarricense. Heredia, Costa Rica: CIDE, Universidad Nacional.

Castro, M., Díaz, M., Fonseca, H., León, A., Moraga, L., Ruiz, S. et al. (2007). II Informe final de investigación. Perfiles, Dinámicas Y Desafíos del Sistema Educativo Formal Costarricense. Heredia, Costa Rica: CIDE, Universidad Nacional.

CONARE. (2006). Hacia un modelo educativo para elevar la calidad de la educación costarricense. San José, Costa Rica: Autor.

CONARE/Programa Estado de la Nación en Desarrollo Humano Sostenible. (2008). Estado de la Educación Costarricense. San José, Costa Rica: Autor.

Decanato-CIDE. (2005). Programa Educación y Desarrollo en Costa Rica. Heredia, Costa Rica: CIDE, Universidad Nacional.

Decanato-CIDE. (2007). Plan Estratégico. Heredia, Costa Rica: CIDE, Universidad Nacional.

Dengo, M. (1997). Universidad y Educación. Foro-Jornadas de Investigación. San José, Costa Rica: Departamento de Publicaciones de la Universidad de Costa Rica.

Román, I. (2009). Implicaciones del contexto socio-cultural para la formación docente. Conferencia dictada en el CIDE, Curso Actualización Pedagógica. CONARE.

Shön, D. (1998). El profesional reflexivo. Barcelona, España: Paidós.

Teichler, U. (2003). The future of higher education and the future of higher education research. Tertiary Education and Management. Vol. 9, 171-185. 